\title{
Oscillation Frequency of Flat Ring of Viscous Capillary Fluid
}

\author{
Alexander D. Gerber*, Evgenii A. Gerber
}

LLC Geomera, Komsomolskaya St. 61, Yekaterinburg, Russia

Corresponding Author Email: evgenii.a.gerber@gmail.com

https://doi.org/10.18280/ijht.370109

Received: 21 September 2017

Accepted: 10 December 2018

\section{Keywords:}

fluid mechanics, flat ring of viscous capillary fluid, ocsillations

\begin{abstract}
The paper considers a problem of a motion of a flat ring of a viscous capillary fluid with free boundaries rotating by inertia. Transformations of system of Navier-Stokes equations to dimensionless variables are described. Numerical method for system «gas-liquid-gas» analysis is applied. Motion mode of studied system whereby ring sizes periodic oscillations were observed was found. Ring size oscillates around fixed point. The fixed point coordinates in case when initial angular velocity is equal to constant was found. It is shown that new dimensionless equations can be presented as equation similar to equation of harmonic dumped oscillator. Such view allows to obtain formula for ring size oscillation frequency.
\end{abstract}

\section{INTRODUCTION}

The paper considers a problem of a motion of a flat ring of a viscous capillary fluid with free boundaries rotating by inertia. Suchlike motion corresponds to the rotation of infinite cylinder of a fluid in an environment without gravity. An analog of studied object is a cross section of vortex rings in case of some restrictions. In example, vortex rings may be observed in a turbulent flow over a plane's wing or when fluid flow out a nozzle. Tornadoes, whirlpools, ring galaxies in a cross section is also similar to studied object. Therefore, the research does not lose its relevance.

The research mainly focuses on periodic mode of movement of studied object.

\section{PLANE PROBLEM STATEMENT}

Plane problem statement consists of Navier-Stokes equations and has the next form:

$$
\begin{gathered}
\frac{\partial \vec{u}}{\partial t}+(\vec{u} \cdot \nabla) \vec{u}-v \Delta \vec{u}-\frac{1}{\rho} \nabla p=0 \\
\nabla \vec{u}=0 \\
T_{1 B \mid 1} \vec{n}_{1}-T_{1 B \mid 2} \vec{n}_{1}=2 \sigma H_{1} \vec{n}_{1} \\
T_{2 B \mid 1} \vec{n}_{2}-T_{2 B \mid 2} \vec{n}_{2}=2 \sigma H_{2} \vec{n}_{2} \\
\left.\vec{u}\right|_{t=0}=\vec{u}_{0}
\end{gathered}
$$

The stress tensor for classical Stokes fluid is

$$
T=-p I+2 \mu D
$$

The strain rate tensor is

$$
D_{i j}=\left(\partial u_{i} / \partial x_{j}+\partial u_{j} / \partial x_{i}\right)
$$

The stress tensor for gas at boundaries is:

$$
T_{i \Gamma}=\left(\begin{array}{cc}
-p_{i g}(t) & 0 \\
0 & -p_{i g}(t)
\end{array}\right)
$$

Geometric problem statement.: Let $r=R_{1}(t), r=R_{2}(t)$ be the outer and inner ring boundaries.

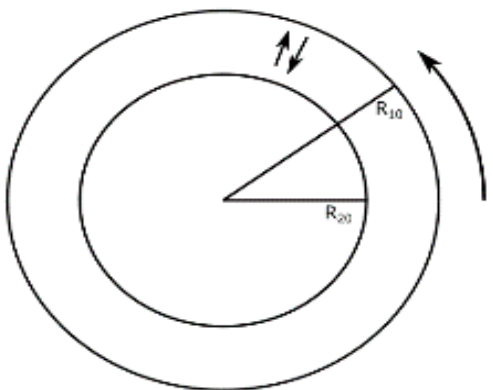

Figure 1. The geometry of the liquid ring $\left(R_{10}, R_{20}>0\right)$

The problem has axial symmetry thus let equations (1) be written in polar coordinates. The $\overrightarrow{u(r)}=\left(u_{r}(r) ; u_{\varphi}(r)\right) ; r \in$ $\left[R_{2}(t) ; R_{1}(t)\right]$ is new sought velocity vector. Let us also apply next dimensionless transformation:

$\left\{\begin{array}{c}\xi(t)=R_{2}^{2}(t) / R_{20}^{2} \\ \eta(t)=\left(r^{2}-R_{2}^{2}(t)\right) / R_{20}^{2}=-\xi+r^{2} / R_{20}^{2} \\ \eta(t) \epsilon[0, a], a=R_{10}^{2} / R_{20}^{2}-1\end{array}\right.$

$\eta(t)$ value range is time invariant. $\eta=a$ corresponds to point on external border, $\eta=0$ on internal.

Then let us use next dimensionless substitutions:

$\left\{\begin{array}{c}\tau=v t / R_{20}^{2} \\ \Psi=u_{r} r / v \\ \omega=u_{\phi} R_{20} / v \sqrt{\xi+\eta}\end{array}\right.$ 
Equations (1) transforms to the next form after applying (5) and (6).

$$
\left\{\begin{array}{c}
\frac{d \Psi}{d \tau} \ln \left(1+\frac{a}{\xi}\right)-\frac{a\left(\Psi^{2}-4 \Psi\right)}{\xi(a+\xi)} \\
-\int_{0}^{a} \omega^{2} d \eta-\int_{0}^{a} \frac{\partial \omega}{\partial \eta} d \eta= \\
-\delta_{1}\left(\frac{1}{\sqrt{\xi}}+\frac{1}{\sqrt{a+\xi}}\right)+\delta_{2}\left(p_{2 g}(\tau)-p_{1 g}(\tau)\right) \\
\Psi(0)=\Psi_{0} \\
\frac{d \xi}{d \tau}=2 \Psi ; \quad \xi(0)=1 \\
\frac{\partial \omega}{\partial \tau}+\frac{2 \Psi \omega}{(\xi+\eta)}=8 \frac{\partial \omega}{\partial \eta}+4(\xi+\eta) \frac{\partial^{2} \omega}{\partial \eta^{2}} \\
\omega(0, \eta)=\omega_{0}(\eta) ;\left.\quad \frac{\partial \omega}{\partial \eta}\right|_{\eta=0, a}=0
\end{array}\right.
$$

where $\delta_{1}=2 R_{20} k_{\sigma} \sigma v^{-2} \rho^{-1}$ and $\delta_{2}=2 R_{20}^{2} \rho^{-1} v^{-2}$ are multipliers that introduced for simplification.

The problem (7) is well-known and were previously investigated [1-2]. There were founded that ring can be in one of three motion modes: infinite expansion, collapse and stationary motion. System of equations (7) has no common analytical solution at the current moment so numerical methods were used for ring behavior analysis. Numerical methods were tested on single known solution [3].

Numerical methods are widespread [4] and have proven themselves well.

\section{RESULTS}

Geometrical and physical characteristics were varied during the numerical experiments. [5] Existence of previously discovered motion modes were confirmed. Except it dumped periodic oscillations of gas-liquid-gas system were found.

Figure 2 illustrates numerical experiment for following inputs:

1. the physical characteristics of the water at room temperature for the liquid ring in air;

2. ring sizes are measured in centimeters $-h=10^{-2} \mathrm{~m}$;

3. initial dimensionless angular velocity $\omega_{0}=C_{0}$, the initial dimensionless radial velocity $\Psi(0)=10$.

Quality factor of oscillation system reduce as 8 times as increasing viscosity as twice as high, thus energy lost as a result of dissipation reduced as 8 times as less.

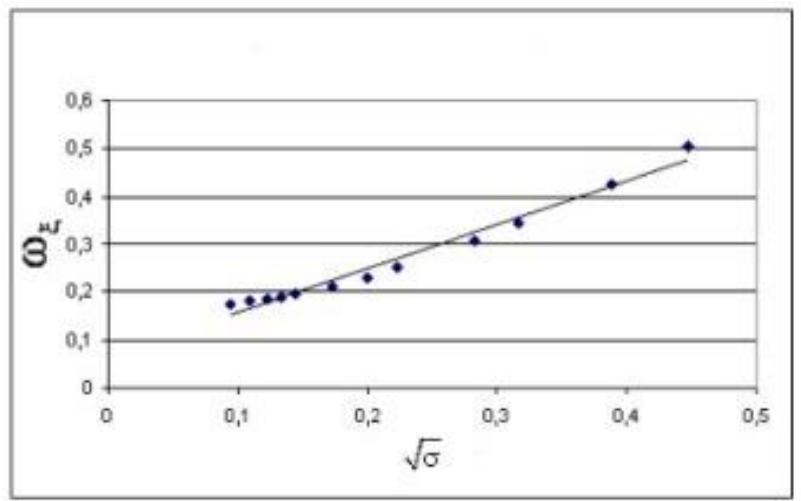

Figure 2. Oscillation frequency dependence on surface tension value

Straight lines are analytical result, dots are results of numerical simulation.
According to experiments we can conclude that oscillations are observed for all functions $\Psi, \xi, \omega$. Their period of oscillations is the same. Thus, let us select only one function for further analysis [6-7]. Function $\xi$ is the most illustrative in our opinion because it directly depends on ring sizes.

System of differential equations (7) has a fixed point with coordinates $\left(\Psi=0, \xi=\xi_{*}, \omega=C_{0}(a+2) /\left(a+2 \xi_{*}\right)\right)$, where $\xi_{*}$ is a solution of nonlinear equation:

$\delta_{1}\left(\frac{1}{\sqrt{\xi}}+\frac{1}{\sqrt{a+\xi_{*}}}\right)=\delta_{2}\left(\frac{p_{20}}{\xi_{*}^{\gamma}}-p_{10}\right)+\frac{a \mathrm{C}_{0}(a+2)^{2}}{\delta_{1}\left(a+2 \xi_{*}\right)^{2}}$

Comparison of fixed points calculated by formula (8) and obtained from numerical simulation get us error about $1 \%$. Thus, it can be concluded that numerical simulation and analytical solution have good consistency.

Further analysis consists of obtaining oscillation characteristics. Assume that integrals of $\omega$ are insignificant. Then excluding $\Psi$ from (7) we get second-order nonlinear ordinary differential equation for $\xi$ :

$\frac{d^{2} \xi}{d t^{2}}+\frac{2}{\ln \left(1+\frac{a}{\xi}\right)}\left(\delta_{1}\left(\frac{1}{\sqrt{\xi}}+\frac{1}{\sqrt{a+\xi}}\right)-\frac{a\left(\xi^{\prime 2}-8 \xi^{\prime}\right)}{d \xi(a+\xi)}-\delta_{2}\left(p_{2 g}(\tau)-\right.\right.$

$\left.\left.p_{1 g}(\tau)\right)\right)=0$

The second member in (9) is a nonlinear function of $\xi$ and $\xi^{\prime}$. So, calculating Taylor's series around fixed point for this member and taking into account only linear approximation we obtain harmonic damped oscillations equation. Formula for the oscillation frequency of the dimensionless inner radius is obtained from the multiplier in front of $\xi$ :

$\omega_{\xi}=\sqrt{\frac{4 \sigma}{\rho R_{20}^{3}}\left(-\alpha_{1} \beta_{2}+\alpha_{2} \beta_{1}\right)+\frac{4}{R_{20}^{2} \rho \xi_{s}^{\gamma}}\left(\frac{\alpha_{1} \gamma}{\xi_{s}}-\alpha_{2}\left(p_{20}-p_{10} \xi_{s}^{\gamma}\right)\right)}$

where $\alpha_{1}=1 / \ln \left(1+a / \xi_{*}\right), \alpha_{2}=a / \xi_{*}^{2} / \ln \left(1+a / \xi_{*}\right), \beta_{1}=$ $1 / \sqrt{\xi_{*}}+1 / \sqrt{a+\xi_{*}}, \beta_{2}=1 / 2 / \sqrt{\xi_{*}^{3}}+1 / 2 / \sqrt{\left(a+\xi_{*}\right)^{3}}$.

Note that suchlike transformations can be also done for $\Psi$ $(\tau)$ and $\omega(\tau, \eta)$

It follows from (10) that oscillation frequency has the next properties in a first approximation:

- Oscillation frequency does not depend on the coefficient of dynamic viscosity $v$

- Oscillation frequency is in proportion to $\sqrt{\sigma}$

- Oscillation frequency is in inverse proportion to and to $\sqrt{\rho}$ and $\sqrt{R_{20}^{3}}$

- Oscillation frequency is nonlinear function of $a$

Obtained formula (10) has good consistency with the results of numerical simulation (Figure 2).

Detailed research of $\Psi$ and $\omega$ behavior is the same as for $\xi$ and can be omitted without loss of generality. But taking into account form of the second equation in (7) we can conclude that the frequency of the periodic variations of these values coincide with $\xi$.

Geometric and physics characteristics that were used in numerical experiments are matched to Reynolds numbers range [1..1000]. 


\section{CONCLUSION}

Research shows that numerical simulation results are coincide with analytical and gives adequate information about studied object behavior.

Using (7) we obtained nonlinear oscillation equation for relative ring sizes and approximation for frequency.

Motion mode in which system comes to steady state through oscillation process were described. Suchlike motion mode is physically expected because system includes forces acting along different directions. Centrifugal force acting in direction out of ring center and surface forces acting in backward direction. Pressure difference on outer and inner borders can acting in both directions.

Important detail is that the ring continues rotating with constant angular velocity after sizes oscillations are stopped.

Assuming all the above, it becomes possible to estimate oscillation frequency of ring sizes depend on geometrical and physical characteristics.

\section{REFERENCES}

[1] Lavrenteva OM. (1978). Unsteady motion of a rotating ring of a viscous capillary fluid, 1978, Dynamics of continuous media: Collection of scientific works. Novosibirsk 31: 52-60.

[2] Lavrenteva OM. (1980). Limit motion modes of viscous rotating ring, 1980, Dynamics of continuous media: Collection of scientific works. Novosibirsk 44: 15-34.

[3] Bytev VO. (1970). Unsteady motion of a viscous incompressible fluid with free boundaries of the ring. $\mathbf{J}$ Appl Mech Tech Phys 11: 432. https://doi.org/10.1007/BF00908073

[4] Wang XR, Ren GL, Zhang JX. (2018). Numerical simulation and optimization analysis of thermal balance of heavy oil box-type substation louver arrangement. Mathematical Modelling of Engineering Problems 5(1): 21-26. https://doi.org/10.18280/mmep.050103

[5] Puhnachev VV. Non-classical problems of boundary layer theory. Novosibirsk.
[6] Andreev VK, Bublik VV, Bytev VO. (2003). Symmetries of nonclassical hydrodynamics models. Novosibirsk.

[7] Annin BD. (1985). The group properties of the equations of elasticity and plasticity. Bytev V.O., Senashev S.I., Novosibirsk, Science.

\section{NOMENCLATURE}

$\vec{u}=\left(u_{x}, u_{y}\right)$
$\nabla$
$\mathrm{t}$
$\rho$
$p$
$\Delta$
$v$
$\sigma$
$T_{i B \mid 1}$
$T_{i B \mid 2}$
$H_{i}$
$n_{i}$
$I$
$\mu$
$D_{i j}$
$T_{i \Gamma}$

$p_{i g}(t)$
$R_{10}$
$R_{20}$
$k_{\sigma}$

vector velocity field

Hamiltonian

Time, $\mathrm{s}$

Density, $\mathrm{kg} / \mathrm{m}^{3}$

Pressure, $\mathrm{Pa}$

Laplace operator

kinematic viscosity coefficient, $\mathrm{m}^{2} / \mathrm{s}$

surface tension coefficient, $\mathrm{N} / \mathrm{m}$

Cauchy stress tensor for liquid on $i$ th border $(i=1 . .2), \mathrm{Pa}$

Cauchy stress tensor for gas on $i$-th border, $\mathrm{Pa}$

mean curvature on $i$-th border $i=1 . .2$, $m^{-1}$

normal on $i$-th ring border $i=1 . .2$

identity tensor

dynamic viscosity, $\mathrm{Pa} S$

strain rate tensor, $s^{-1}$

stress tensor of environment which in contact with liquid on $i$-th border $i=1$..2, $\mathrm{Pa}$

the variation law of gas pressure on $i$-th border $i=1 . .2, \mathrm{~Pa}$

Outer ring border initial position, $\mathrm{m}$ Inner ring border initial position, $\mathrm{m}$ Coefficient of presence or absence of surface tension. $k_{\sigma}$ can be equal to 0 or to 1 